\title{
Enhancing Effects of Post-Learning Stress on Memory
}

\author{
Mingming Lin ${ }^{1,2}$, Yoshihiko Tanno ${ }^{2}$ \\ ${ }^{1}$ Research Fellow of the Japan Society for the Promotion of Science, Tokyo, Japan \\ ${ }^{2}$ Department of Life Sciences, Graduate School of Arts and Sciences, The University of Tokyo, Tokyo, Japan \\ Email: mingming@10.alumni.u-tokyo.ac.jp
}

Received February $23^{\text {rd }}, 2012$; revised April 26 ${ }^{\text {th }}, 2012$; accepted May $29^{\text {th }}, 2012$

\begin{abstract}
To investigate the enhancing effect of post-learning stress on memory, we requested 38 Japanese undergraduates to perform a learning task that involved positive, negative, and neutral words with controlled arousal and subsequently assigned them to a stress group (exposed to acute white noise) or a control group. After a 10-min filler task, we administered a delayed free recall test and a recognition test. We found that exposure to acute stress after learning significantly enhanced recognition memory of words, but found no differences in memory scores for stimuli of varying valence. We accordingly propose that post-learning stress, though enhancing memory performance, may not depend on word valence when stimulus arousal is controlled. This is the first study to find that post-learning stress enhances memory after a short delay, and it has several implications with regard to traumatic memories in stress-related disorders.
\end{abstract}

Keywords: Post-Learning Stress; Memory; Valence; Arousal

\section{Introduction}

Stressful events are remembered well, and are particularly vivid for individuals with stress-related disorders, such as post-traumatic stress disorder (PTSD) (Berntsen, Willert, \& Rubin, 2003). Researchers investigating laboratory stress have recently reported that people remember well not only the memory of a stressor itself but also the memory that is created immediately before or after stress. This effect was thought to be stress-enhanced memory consolidation (Wolf, 2008). However, there are some incongruities in different studies in the effect of acute stress on emotional memory and neutral memory.

With regard to the effect of stress on memory consolidation, many studies have found that stress after learning (i.e., postlearning stress) can enhance memory performance. These studies have used stimuli, such as words (Smeets, Otgaar, Candel, \& Wolf, 2008), pictures (Cahill, Gorski, \& Le, 2003; Yonelinas, Parks, Koen, Jorgenson, \& Mendoza, 2011; Preuss \& Wolf, 2009), or a short film or story (Andreano \& Cahill, 2006; Beckner, Tucker, Delville, \& Mohr, 2006). In contrast, other studies have found that stress before learning (i.e., pre-learning stress) both enhances and impairs memory. For instance, Kirschbaum, Wolf, May, Wippich, and Hellhammer (1996) found that pre-learning stress impaired memory, while some researchers have reported that such pre-learning stress tended to impair neutral memory while either enhancing or having no influence on positive and negative emotional memory (Jelicic, Geraerts, Merckelbach, \& Guerrieri, 2004; Smeets, Jelicic, \& Merckelbach, 2006). Studies on pre-learning stress have found that stress affords greater benefits to emotional memory than to neutral memory; however, the observed effects of pre-learning stress may have been caused by the mixed influence of stress on the initial encoding and the consolidation of learning materials (Schwabe, Wolf, \& Oitzl, 2010). Therefore, the use of a post-learning manipulation appears to be desirable to determine the effect of stress on memory consolidation.
However, there is a controversy in studies of post-learning stress as to whether acute stress enhances neutral or emotional memories. Some previous studies have reported that acute stress selectively enhances only negative, not neutral, memories, after using both negative emotional and neutral materials (Cahill et al., 2003; Smeets et al., 2008). Others have reported an enhancement of neutral memories after using both negative and neutral materials (Yonelinas et al., 2011), or relatively neutral materials alone (Andreano \& Cahill, 2006; Beckner et al., 2006). However, to determine whether post-learning stress enhances emotional or neutral memory, the enhancing memory effect should be investigated using three-valence materials (neutral, negative, and positive). The use of positive material should be considered to prevent the mood-congruence effect (i.e., people retrieve more negative memories in response to a negative mood that may have been induced by stress manipulation; Blaney, 1986). Preuss and Wolf (2009) used positive, negative, and neutral pictures and reported that post-learning stress enhanced the recall of neutral, but not emotionally positive or negative, items. This result is incongruent with those of previous studies that found that post-learning stress had an enhancing effect on negative memory. It remains unclear as to whether post-learning stress, which is thought to affect memory consolidation, has a greater enhancing effect on emotional memory or on neutral memory.

A possible limitation of previous studies is that they followed the traditional protocol in which participants were exposed to acute stress after a learning task, followed by a long delay (hours to days) before the memory test was administered. To the best of our knowledge, no study has investigated whether post-learning stress affects memory after a shorter delay. Furthermore, in previous studies, participants spent most of this period outside the laboratory. Therefore, a concern with most studies is that the participants may have been influenced by some stressful events after leaving the laboratory and that these events may have had a greater effect on memory than did the 
stress manipulation they experienced in the laboratory setting. This uncontrollable influence may also have caused the incongruence in these studies' findings on stress effects.

In an attempt to overcome the aforementioned shortcomings and resolve the controversies in the literature, the present study uses three-valence (positive, negative, and neutral) stimuli, while conducting all procedures involving the delayed memory test within a controlled laboratory environment, in order to investigate whether post-learning acute stress enhanced emotional memory or neutral memory. To the best of our knowledge, this is the first study to test the post-learning stress effect following a short delay after the administration of the stressor. Furthermore, we used two-character compound words as learning materials in our memory task. Each word consists of two Chinese characters, and thus, all words were of uniform length; this condition circumvented the possibility of memory impairment due to the word-length effect (Baddeley, Thomson, \& Buchanan, 1975) which might influence the effect of acute stress on memory performance. We expected that our new protocol would eliminate the uncertainty regarding the influence of extraneous variables, which was seen in previous studies.

\section{Methods}

\section{Participants}

We randomly assigned 38 Japanese undergraduate students (18 females and 20 males), with a mean age of 19.2 years $(S D=$ 1.42 , range: $18-26)$, to a stress group $(n=19$, nine females and ten males, mean age: $19.0, S D=0.94$, range: $18-21$ ) or a control group $(n=19$, nine females and ten males, mean age: 19.5, $S D=1.78$, range: $18-26$ ). The study was approved by the local ethics committees of the University of Tokyo. All the participants gave written informed consent before the experiment began and were well debriefed on completion of the study.

\section{Materials and Apparatus}

The target stimulus list in the learning task comprised 24 two-character compound words, which included eight neutral words, eight positive words, and eight negative words. All words were chosen from the list provided by Gotoh and Ohta (2001), who investigated the affective valence, frequency of use, ease of learning, and imagery of two character compound words. Furthermore, to prevent the serial position effect, two series of three filler words, which had neutral, negative, and positive valence, were each placed before and after the targets. Arousal of these words was investigated in preliminary research $(n=18)$. A one-way analysis of variance (ANOVA) showed that all three word categories (neutral, positive, and negative) differed significantly with regard to valence (neutral: $M=3.73$, $S D=0.05$; positive: $M=1.95, S D=0.17$; negative: $M=5.99$, $S D=0.24$; on a 7-point scale, ranging from 1 ("extremely positive") to 7 ("extremely negative"); $F(2,21)=1120.81, p$ $<.001$ ). The score of arousal did not differ significantly (neutral: $M=4.06, S D=0.45$; positive: $M=4.24, S D=0.44$; negative: $M=4.25, S D=0.36$; on a 7 -point scale ranging from 1 ("extremely low arousal") to 7 ("extremely high arousal"); $F$ (2, $21)=0.53, p=.60$ ). Furthermore, no differences were found in the frequency of use, ease of learning, and imagery; all $p \mathrm{~s}$ $>.10$.

For the recognition test, another 24 "new" words were chosen from the same list. These words were similar to the "old" words, which were presented in the learning task. A 2 (old vs. new) $\times 3$ (neutral vs. positive vs. negative) ANOVA showed that the new words did not differ significantly from the old words with regard to valence, arousal, frequency of use, ease of learning, and imagery; all $p s>.10$.

All stimuli were presented at the center of a 12.1-inch black computer screen in a 60-point white Gothic font. The entire experiment was programmed in MATLAB 7.0.4, using the Psychophysics Toolbox extensions (Brainard, 1997; Pelli, 1997).

\section{Subjective Stress Measure}

Subjective stress was measured using the Japanese version of the Profile of Mood State Brief Form (POMS-BF; Yokoyama, 2005). POMS is a widely used self-report measure for typical and persistent mood reactions to current situations. POMS-BF comprises 30 items across six subscales: Tension-Anxiety, Depression-Dejection, Anger-Hostility, Fatigue, Confusion, and Vigor. The participants indicated the extent to which they agreed with adjectives describing their current mood or feelings, on 5-point scales (0: "not at all"; 4: "extremely"). The subjective stress scores were calculated using the following formula: Tension-Anxiety + Depression-Dejection + Anger-Hostility + Fatigue + Confusion - Vigor.

\section{Stress Manipulation}

Participants in the stress group were exposed to $80-\mathrm{dB}$ white noise through a headset for $5 \mathrm{~min}$ continuously. Noise stress is a stress manipulation that has been widely used in previous studies (e.g., Carter \& Beh, 1989; Smith, Whitney, Thomas, Perry \& Brockman, 1997), and known to affect cardiovascular function such as blood pressure. Noise stress allows us to expose participants to acute stress during a task, without interrupting their operation of that task.

\section{Procedure}

After filling out the consent form, participants were instructed to perform several computer tasks while wearing headphones. They were informed that they might hear some sound from the headphones while performing these tasks. Initially, participants performed a learning task, in which the word stimulus was presented at the center of the computer screen for $2000 \mathrm{~ms}$, after a fixation cross was presented for $500 \mathrm{~ms}$. The inter-trial interval was $1000 \mathrm{~ms}$. The presentation order of both the 24 target words and two series of filler words were randomized.

Immediately after the learning task, the participants performed a 5-min calculation filler task, in which stimuli were also presented on a computer screen. In this task, the participants were required to press keys on the keyboard to give their answers. Those in the stress group were exposed to $80-\mathrm{dB}$ white noise during this task, while the control group was not exposed to any sound. Using POMS, we measured subjective stress before and after this filler task.

After another 10-min filler task, the participants performed a delayed free recall task followed by a recognition task, in which the 48 word stimuli were randomly presented at the center of a computer screen, in a similar manner to the learning task, for a maximum of $2000 \mathrm{~ms}$, or until the participant responded. When 
each word was shown, the participant was required to decide whether the word was "old" or "new" by pressing the "F" or "J" key, respectively, within $2000 \mathrm{~ms}$. The experiment lasted for approximately $60 \mathrm{~min}$, and all participants were tested individually.

\section{Results}

\section{Subjective Stress}

Subjective stress was indexed by an increase in the scores of POMS (increase $=$ POMS before stress/control task-POMS after stress/control task). The mean subjective stress scores are shown in Figure 1. A two-sample $t$-test showed that the participants in the stress group had higher subjective stress than those in the control group (stress group: $M=8.47$, control group: $M=-2.00), t(36)=4.16, p<.001$ (two-tailed)). The results showed that the stress manipulation was effective.

\section{Memory Performance: Delayed Recall}

Numbers of correctly-recalled target items were added up separately for neutral, positive, and negative words, respectively. The proportion of correct recall was calculated by dividing correctly-recalled numbers by the total amount of presented target-word numbers for each valence (neutral: $M=.26$, positive: $M=.24$, negative: $M=.24$ in the stress group; neutral: $M=.18$, positive: $M=.21$, negative: $M=.18$ in the control group). Recall performance was analyzed using a 2 (Group: stress group vs. control group) $\times 3$ (Valence: neutral vs. positive vs. negative) ANOVA with valence as a repeated factor. The ANOVA showed that delayed recall score in the stress group $(M=.24, S D=.14)$ tended significantly higher than in the control group $(M=.19, S D=.13)$, as demonstrated by a tendency of significant main effect of Group, $F(1,36)=3.01$, $p$ $=.09$. No other significant main effects of Valence, $F(2,72)=$ $0.11, p=.89$, or Group $\times$ Valence interaction, $F(2,72)=0.44, p$ $=.65$, were detected.

\section{Memory Performance: Recognition}

Recognition score was calculated as the discrimination (hit rate, proportion of old items correctly recognized, minus false alarms rate, proportion of new items falsely identified as old). A higher recognition score indicates enhanced recognition performance. Mean recognition performance is shown in Figure 2. Recognition performance was analyzed using a 2 (Group: stress group vs. control group) $\times 3$ (Valence: neutral vs. positive vs. negative) ANOVA, with valence as a repeated factor. The ANOVA yielded a significant main effect of Group, $F(1,36)=$ $5.42, p<.05$, and of Valence, $F(2,72)=3.50, p<.05$. The main effect of Group showed that the total score of recognition in the stress group $(M=.68, S D=.20)$ was significantly higher than that in the control group $(M=.56, S D=.26)$, regardless of valence. Bonferroni corrected post hoc analyses on Valence showed that the recognition performance of positive words tended significantly higher than that of negative words ( $p$ $=.08$ ). However, no significant interactive effects, $F(2,72)=$ $1.61, p=.21$, were detected.

\section{Discussion}

The main purpose of this study was to examine the enhanc-

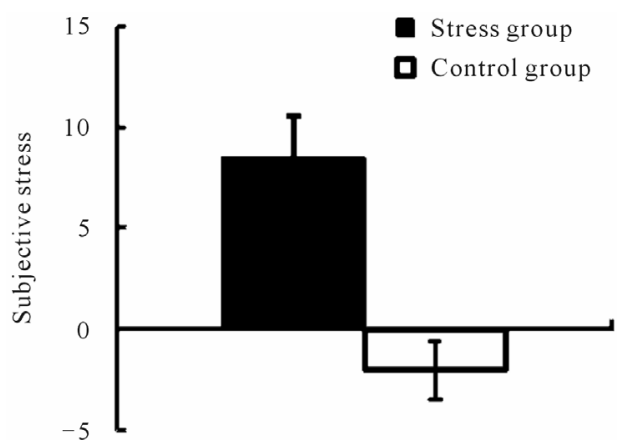

Figure 1.

Mean subjective stress scores for both the stress group and the control group. Error bars indicate $\pm 1 S E$.

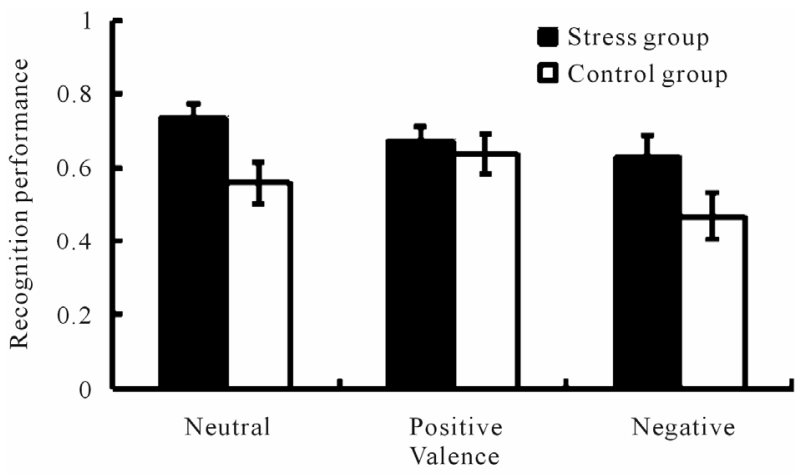

Figure 2.

Mean recognition performance as a function of valence and groups. Error bars indicate $\pm 1 S E$.

ing effect of post-learning stress on the memory of words. It was the first investigation to test post-learning stress effect in a short time delay, and used uniform-length words of three valences (positive, negative and neutral) as learning material to prevent word-length effect. We found that acute white noise stress enhanced memory performance significantly in a recognition test, regardless of valence.

Participants in the stress group exhibited a significant subjective stress response following exposure to white noise. With regard to the effect of acute stress on word memory performance, the results suggest that exposure to acute stress after learning significantly enhanced recognition memory, and tended to significantly enhance delayed recall memory. This finding of an effect of enhancing memory is in line with previous studies. Furthermore, recognition performance tended higher for positive than for negative words, regardless of groups. The higher positive memory is thought to result from a positive bias, where healthy people prefer to have better memory of positive things than of negative ones (Matlin \& Stang, 1978).

We could not find differences in memory scores for positive, negative, and neutral word stimuli. There was no stress-caused selective enhancement of emotional memory or neutral memory. This result is incongruent with previous research, in which selective enhancement of emotional negative memory (Cahill et al., 2003; Smeets et al., 2008) or neutral memory (Preuss \& Wolf, 2009; Yonelinas et al., 2011) was reported. One possible explanation for this result is that post-learning stress enhances word memory performance, but relies on material arousal and 
not on the valence of stimuli. Both the valence and arousal of the material mediate the processing of emotional memory (Lang, Newhagen, \& Reeves, 1996), and arousal ranges from calming to exciting, while valence ranges from positive to negative. In examining the relationship between the effect of acute stress on emotional memory, especially pre-learning stress and acute stress on memory retrieval, Wolf (2009) asserted that arousal might be more important than valence with regard to the occurrence of stress effects. Stress is much more likely to selectively affect the memory of high arousing material than low arousing material. We controlled the arousal level of learning words stimuli used in this study, so this effect of acute stress on material arousal might have caused a null effect between neutral and emotional memory performance. Therefore, there is a possibility that the effect of stimulus arousal may have been responsible for the incongruent results of previous studies regarding whether post-learning stress enhances memory of emotional stimuli (Cahill et al., 2003; Smeets et al., 2008) or neutral stimuli (Preuss \& Wolf, 2009; Yonelinas et al., 2011). In other words, using highly arousing emotional stimuli would enhance emotional memories owing to acute stress, while using emotional and neutral stimuli of the same arousal level (or perhaps more highly arousing neutral stimuli) would enhance neutral memory.

However, in the present study, the enhancing effect on memory observed in the stress group was only a non-significant tendency with regard recall performance, but was significant with regard to recognition performance. In previous studies reporting an enhancing effect in recall performance using word stimuli, the percentage of correct stem cued recall was above $50 \%$ (Smeets et al., 2008), and in those that reported using free recall of slide stimuli, the correct recall performance was over $37 \%$ (Cahill et al., 2003). In the current study, the total recall percentage across all participants was only $22 \%$. One potential explanation for this is that the free recall test was too difficult; consequently, the effect of acute stress on recall would have been hard to observe, owing to the floor effect. Another reason could be that the enhancing effect of stress may have appeared in the recognition test, which was administered after the delayed recall test. This finding of enhanced recognition is in accordance with the results of Yonelinas et al. (2011), who also administered a recognition test after a free recall test.

Despite the scope for further research in this area, the present paper - to the best of our knowledge - is the first to show an enhancing effect of post-learning stress following only a short, 10-min delay after the administration of the stressor. With regard to the mechanisms of post-learning stress, previous studies have stated that stress modulates memory consolidation so as to enhance memory performance; however, it is not clear whether stress effects reflect the "systems" or "synaptic" forms of consolidation. Yonelinas et al. (2011) stated that synaptic forms might lead to an enhancing effect of post-learning stress two hours after the administration of the stress. Our finding of enhancement after a short 10-min delay may substantiate the theory of synaptic consolidation.

Evidence of the effect of stress on memory would be expected to improve understanding of memory consolidation with regard to stress-related disorders such as PTSD. On the basis of the evidence of enhanced memory consolidation as a result of stress, Schwabe et al. (2010) have postulated that the extreme arousal associated with a traumatic event leads to "over-consolidation." Our findings suggest that because the enhancing effect of stress on memory consolidation would start shortly after learning, administering a treatment intended to prevent consolidation, immediately after a stressful event, may help patients overcome traumatic memories.

Finally, some limitations of this study should be mentioned. First, we used only arousal-controlled words, that is, words that elicit little emotional arousal. To determine the effect of material arousal, further studies that use both low- and high-arousal three-valence learning materials should be conducted. Second, it remains unclear whether acute stress directly affects recognition memory, or whether the recalled memory, which was affected by stress, indirectly affects recognition memory. Additional investigations should use only a recognition test to provide answers. Finally, it is unclear whether the enhancement of memory after a short time delay in this study will be sustained for a long time, as reported by previous studies where long-time delays (hours to days) were considered. Further research that compares short and long time delays is necessary and will proffer some evidence in response to this question.

In summary, using positive and negative emotional materials and neutral materials, we found that the administration of acute noise stress after a learning task enhanced memory performance of words after a short delay. However, in this study, there was no selective enhancement among negative, positive, and neutral memory. The arousal level of the learning material may be responsible for this result. This finding suggests that at least in some conditions, post-learning stress will enhance memory performance regardless of the valence of memory materials.

\section{Acknowledgements}

We would like to thank Hironori Akechi and Masanori Kobayashi for their helpful comments on the manuscript.

\section{REFERENCES}

Andreano, J. M., \& Cahill, L. (2006). Glucocorticoid release and memory consolidation in men and women. Psychological Science, 17, 466-470. doi:10.1111/j.1467-9280.2006.01729.x

Baddeley, A.D., Thomson, N., \& Buchanan, M., (1975). Word length and the structure of short-term memory. Journal of Verbal Learning and Verbal Behavior, 14, 575-589.

doi: 10.1016/S0022-5371(75)80045-4

Beckner, V. E., Tucker, D. M., Delville, Y., \& Mohr, D. C. (2006). Stress facilitates consolidation of verbal memory for a film but does not affect retrieval. Behavioral Neuroscience, 120, 518-527. doi: $10.1037 / 0735-7044.120 .3 .518$

Berntsen, D., Willert, M., \& Rubin, D. C. (2003). Splintered memories or vivid landmarks? qualities and organization of traumatic memories with and without PTSD. Applied Cognitive Psychology, 17, 675693. doi: $10.1002 / \mathrm{acp} .894$

Blaney, P. H. (1986). Affect and memory: A review. Psychological Bulletin, 99, 229-246. doi:10.1037/0033-2909.99.2.229

Brainard, D.H. (1997). The Psychophysics Toolbox, Spatial Vision, 10, 433-436. doi:10.1163/156856897X00357

Cahill, L., Gorski, L., \& Le, K. (2003). Enhanced human memory consolidation with post-learning stress: Interaction with the degree of arousal at encoding. Learning \& Memory, 10, 270-274. doi: $10.1101 / 1 \mathrm{~m} .62403$

Carter, N. L., \& Beh, H., C. (1989). The effect of intermittent noise on cardiovascular functioning during vigilance task performance. Psychophysiology, 26, 548-559. doi:10.1111/j.1469-8986.1989.tb00708.x

Gotoh, F., \& Ohta, N. (2001). Affective valance of two-compound kanji words. Tsukuba Psychological Research, 23, 45-52. 
Jelicic, M., Geraerts, E., Merckelbach, H., Guerrieri, R. (2004). Acute stress enhances memory for emotional words, but impairs memory for neutral words. International Journal of Neuroscience, 114, 1343-1351. doi:10.1080/00207450490476101

Kirschbaum, C., Wolf, O. T., May, M., Wippich, W., \& Hellhammer, D. H. (1996). Stress- and treatment-induced elevations of cortisol levels associated with impaired declarative memory in healthy adults. Life Sciences, 58, 1475-1483. doi:10.1016/0024-3205(96)00118-X

Lang, A., Newhagen, J., \& Reeves, B. (1996). Negative video as structure: Emotion, attention, capacity, and memory. Journal of Broadcasting \& Electronic Media, 40, 460-477. doi:10.1080/08838159609364369

Matlin, M. W., \& Stang, D. J. (1978). The Pollyanna principle. Selectivity in language, memory, and thought. Cambridge, MA: Schenkman.

Pelli, D. G. (1997). The VideoToolbox software for visual psychophysics: Transforming numbers into movies, Spatial Vision, 10, 437442. doi:10.1163/156856897X00366

Preuss, D., \& Wolf, O. T. (2009). Post-learning psychosocial stress enhances consolidation of neutral stimuli. Neurobiology of Learning and Memory, 92, 318-326. doi:10.1016/j.nlm.2009.03.009

Schwabe, L., Wolf, O. T., \& Oitzl, M. S. (2010). Memory formation under stress: Quantity and quality. Neuroscience and Biobehavioral Reviews, 34, 584-591. doi:10.1016/j.neubiorev.2009.11.015

Smeets, T., Jelicic, M., \& Merckelbach, H. (2006). The effect of acute stress on memory depends on word valence. International Journal of Psychophysiology, 62, 30-37. doi:10.1016/j.ijpsycho.2005.11.007

Smeets, T., Otgaar, H., Candel, I., \& Wolf, O. T. (2008). True or false? Memory is differentially affected by stress-induced cortisol elevations and sympathetic activity at consolidation and retrieval. Psychoneuroendocrinology, 33, 1378-1386. doi:10.1016/j.psyneuen.2008.07.009

Smith, A., Whitney, H., Thomas, M., Perry, K., \& Brockman, P. (1997). Effects of caffeine and noise on mood, performance and cardiovascular functioning. Human Psychopharmacology, 12, 27-33. doi:10.1002/(SICI)1099-1077(199701/02)12:1<27::AID-HUP827>3. 3.CO;2-P

Wolf, O. T. (2008). The influence of stress hormones on emotional memory: Relevance for psychopathology. Acta Psychologica, 127, 513-531. doi:10.1016/j.actpsy.2007.08.002

Wolf, O. T. (2009). Stress and memory in humans: Twelve years of progress? Brain Research, 1293, 142-154. doi:10.1016/j.brainres.2009.04.013

Yokoyama, K. (2005). POMS tansyukuban tebiki to jireikaisetu [The guide and example commentary for POMS brief form]. Tokyo, Japan: Kanekosyobo.

Yonelinas, A. P., Parks, C. M., Koen, J. D., Jorgenson, J., \& Mendoza, S. P. (2011). The effects of post-encoding stress on recognition memory: Examining the impact of skydiving in young men and women. Stress, 14, 136-144. doi:10.3109/10253890.2010.520376 